\title{
FLUTUAÇÃO POPULACIONAL E ANÁLISE FAUNÍSTICA DE CARABIDAE E STAPHYLINIDAE (COLEOPTERA) EM JABOTICABAL, SÃO PAULO
}

\author{
F.J. Cividanes'; T.M.S. Cividanes ${ }^{2}$
}

${ }^{1}$ Universidade Estadual Paulista, Faculdade de Ciências Agráriase Veterinárias, Departamento deFitossanidade, Jaboticabal, SP, Brasil. E-mail fjcivida@fcav.unesp.br

\section{RESUMO}

No presente estudo efetuou-se um levantamento populacional de adultos de Carabidae e Staphylinidae (Coleoptera) visando-se analisar suas populações por meio de índices faunísticos e determinar a influência da temperatura, umidade relativa e precipitação pluvial. O estudo foi realizado em duas áreas com culturas de soja/milho cultivadas em sistemas de plantio direto e convencional adjacentes a fragmento florestal e povoamento dePinus, respectivamente. Os besouros foram amostrados por meio de armadilhas de solo distribuídas em dois transectos de $100 \mathrm{~m}$ de comprimento. A partir dos dados de riqueza e abundância foram calculados índices de diversidade $(\mathrm{H})$, equitabilidade (J), abundância, dominância, freqüência e constância. A influência da temperatura do ar, umidade relativa e precipitação pluvial na ocorrência destes besouros foi analisada por regressão múltipla com seleção de variáveis pelo método stepwise. Os carabídeos Abaris basistriatus Chaudoir, Odontochila nodicornis (Dejean) e Scarites sp. foram dominantes nas duas áreas estudadas. Já Selenophorus alternans Dejean, apenas na área sob sistema de plantio convencional enquanto Megacephala sp. eSelenophorus seriatoporusPutz., em sistema de plantio direto. As espécies dominantes apresentaram apenas um pico populacional por ano, ocorrendo geralmente de dezembro a janeiro. A precipitação pluvial influiu positiva e significativamente na ocorrência de carabídeos com $O$. nodicornis e Megacephala sp. sendo os mais exigentes quanto à umidade para se dispersarem.

PALAVRAS-CHAVE: Controle biológico, condições meteorológicas, Glycine max, Pinus, Zea mays.

\section{ABSTRACT}

FAUNISTIC ANALYSIS AND POPULATIONAL FLUCTUATION OF CARABIDAE AND STAPHYLINIDAE (COLEOPTERA) IN JABOTICABAL, STATE OF SÃO PAULO, BRAZIL. In this study a population survey of Carabidae and Staphylinidae (Coleoptera) adults was carried out with the aim to analyze their populations through faunistic indexes and to determine the influence of temperature, relative humidity and rainfall. The study was conducted in two areas cultivated with soybean/corn under no-tillage and conventional tillage systems and adjacent to a forest fragment and a Pinus stand, respectively. Beetles were sampled by pitfall traps distributed in two $100-m$ transects. The fauna was characterized by diversity $(\mathrm{H})$, evenness $(\mathrm{J})$, abundance, dominance, frequency and constancy indexes. The influence of air temperature, relative humidity and rainfall was verified using stepwise regression. The carabids Abaris basistriatus Chaudoir, Odontochila nodicornis (Dejean) and Scarites sp. were classified as predominant species in both areas, while Selenophorus alternans Dejean only classified as predominant in the area underconventional tillage system, likewise for Megacephala sp. and Selenophorus seriatoporus Putz. in the no-tillage area. The predominant species showed just one population peak eachyear, usually observed from December to January. The pluvial precipitation influenced the occurrence of the carabids positively and significantly, with O. nodicornis and Megacephala sp. being the most demanding species in relation to the effect of humidity on their dispersal.

KEY WORDS: Biological control, Glycine max, Pinus, weather, Zea mays.

\section{INTRODUÇÃO}

A atividade agrícola tem simplificado e fragmentado o ambiente em um mosaico de áreas naturais e áreas modificadas pelo homem. Como resultado, hábitats naturais de insetos predadores têm sido reduzidos a pequenos fragmentos (PFIFFNER; LUKA, 2000). Ao passo que tem sido reportado considerável

\footnotetext{
${ }^{2}$ Pólo Centro Leste, Ribeirão Preto, SP, Brasil.
} 
aumentona freqüência dedeslocamento decarabídeos e outros artrópodes predadores entre as culturas e hábitats adjacentes. Consequentemente, o aumento da abundância desses organismos nas culturas pode contribuir para o controlebiológico de pragas (ASTERAKI et al., 1995; КROMP, 1999).

A ocorrência deespécies decarabídeos nos h ábitats é muito influenciada pelas condições ambientais (Holland, 2002). A ação de fatores bióticos e abióticos foi investigada por THIELE (1977), que indicou como mais importantes a temperatura, a umidade, a luz e características do substrato, destacando-se a umidade do solo (LuF, 1996). Outros fatores também importantes são: disponibilidade de alimento, cobertura vegetal e ciclo de vida (Löver; SundERLAND, 1996; KROMP, 1999).

Muitas espécies de carabídeos e estafilínideos são associadas ao solo de culturas agrícolas (SOTHERTON, 1984). Devido à natureza predatória, muitas espécies destacam-se como agentes de controle biológico de pragas (Clark, 1994; Menalledet al., 1999). Apesar do enorme potencial que apresentam para controlar pragas, no Brasil são escassos os estudos relacionados com esses besouros em culturas e fragmentos florestais. A ocorrência de carabídeos foi relatada nas culturas de eucalipto, algodão, soja, feijoeiro e hortaliças (ZANUnCio et al., 1993; Didonet et al., 1998; THOMAZINI; Thomazini, 2001; CividAnes et al., 2003, CAIXETA et al., 2005; BARros et al., 2006). Os estafilínideos foram citados em estudos de levantamento populacional nas culturas de soja, cacau, goiaba e eucalipto (ZANuncio et al., 1993; Cividanes et al., 1996; GAlli; RAMPAZZO, 1996; BARBOSA; FonseCA, 2002).

Os estudos relacionados com levantamento populacional de insetos possibilitam caracterizar a comunidade estudada por meio deíndices faunísticos (Freitas et al., 2002), os quais permitem determinar espécies dominantes e avaliar o impacto ambiental, tendo por base espécies de insetos como indicadores ecológicos (Silveira Neto et al., 1995). Possibilitam também a obtenção da flutuação populacional das espécies estudadas, fundamental para o entendimento da dinâmica populacional e o desenvolvimento de programas de manejo de pragas (DENT, 1991).

Na presente pesquisa efetuou-se um levantamento populacional de adultos de carabídeos e estafilinídeos em áreas de plantio de soja e milho adjacentes a fragmento florestal e povoamento de Pinus visando-se analisar suas populações por meio de índices faunísticos e determinar a influência da temperatura, umidade relativa e precipitação pluvial.

\section{MATERIAL E MÉTODOS}

O estudo foi conduzido na área experimental da Fazenda de Ensino, Pesquisa e Produção da Faculda- de de Ciências Agrárias e Veterinárias (FCAV), Universidade Estadual Paulista (UNESP) $\left(48^{\circ} 18^{\prime} 58^{\prime \prime} \mathrm{W}\right.$, $21^{\circ} 15^{\prime} 22^{\prime \prime S}$ ) no Município de Jaboticabal, SP. A amostragem foi realizada de 15 de dezembro de 2003 a 30 de novembro de 2005, abrangendo duas safras agrícolas, com coletas quinzenais; eduas entressafras, com coletas mensais, perfazendo o total de 36 datas de amostragem.

Os coleópteros foram amostrados em duas áreas experimentais distantes cerca de $2 \mathrm{~km}$ entre si, apresentando solo tipo Latossolo Vermelho Distrófico textura argilosa. Uma dessas áreas é coberta por 40 ha de cultivo de soja e milho, em sistemas de rotação de culturas e de plantio direto, ficando em pousio na entressafra. Adjacente ao cultivo, há um fragmento de Floresta Tropical Latifoliada Semidecídua. A segunda área experimental constitui-se em campo de 12 ha de plantio convencional de soja e milho conduzido em sistema de rotação, ficando em pousio na entressafra. Adjacente a esse campo há uma área de 3 ha de Pinus caribaea var. hondurensis (Sénécl) Barr. \& Golf. próxima a um povoamento de 5 ha deEucalyptus citriodora Hook, ambos com cerca de 28 anosde idade, sem a ocorrência de desbaste de plantas.

Os insetos foram amostrados por meio de armadilhas de solo (pitfall traps). Cada armadilha era constituída por um copo plástico com $8 \mathrm{~cm}$ de diâmetro e $14 \mathrm{~cm}$ de altura, contendo $1 / 3$ de uma solução de formaldeído à $1 \%$, acrescido de algumas gotas de detergente doméstico. Para a instalação da armadilha foi enterrado a $1 \mathrm{~cm}$ abaixo da superfície do solo, um cilindro de PVC com $8,5 \mathrm{~cm}$ de diâmetro e $20 \mathrm{~cm}$ de altura. Uma cobertura removível de plástico foi colocada sobre cada armadilha, com altura suficiente para permitir a captura de insetos e evitar inundação pela chuva e entrada de restos vegetais. As armadilhas permaneceram instaladas durante sete dias. Após esse período foram encaminhadas ao laboratório para triagem, montagem e identificação dos insetos.

O delineamento amostral foi adaptado de acordo com a metodologia utilizada por BEDFORD; UsHER(1994). Em cada área amostrada foram instaladas dois transectos paralelos de armadilhas de solo, separados 10 metros entre si. Cada transecto apresentou 100 $\mathrm{m}$ de comprimento, sendo $50 \mathrm{~m}$ no campo de cultura anual e $50 \mathrm{~m}$ no fragmento florestal e no povoamento de Pinus. As armadilhas foram instaladas a cada 10 m a partir da borda da cultura e fragmento florestal/ Pinus, perfazendo o total de 24 armadilhas nos dois transectos.

Os dados foram analisados através do software Anafau, desenvolvido no Departamento de Entomologia, Fitopatologia e Zoologia Agrícola da ESALQ/USP obtendo-se valores de dominância, abundância, freqüência, constância e índices de 
equitabilidade (E) e de diversidade de ShannonWeaner $\left(\mathrm{H}, \log _{\mathrm{e}}\right)$. As espécies consideradas dominantes foram as mais abundantes e que também obtiveram os maioresíndices faunísticos de freqüência, constância e dominância (Silveira Neto et al., 1995).

A flutuação populacional foi considerada para as espécies classificadas como dominantes na análise faunística. A influência de fatores meteorológicos foi avaliada por meio de análise de regressão múltipla com seleção de variáveis pelo métodostepwise (DRAPER; SMITH, 1981). Os fatores meteorológicos considerados foram: temperaturas $\left({ }^{\circ} \mathrm{C}\right)$ máxima, mínima e média, umidade relativa (\%) e precipitação pluvial (mm), registrados pela Estação Agroclimatológica da FCAV/ UNESP. Para os fatores meteorológicos temperatura e umidade relativa utilizou-se a média mensal, enquanto para precipitação pluvial considerou-se a soma de precipitação registrada no mês.

\section{RESULTADOS E DISCUSSÃO}

O total de 18 espécies de carabídeos e três espécies de estafilinídeos foram capturadas tanto na área sob sistema de plantio convencional quantona conduzida sob plantio direto (Tabelas 1 e 2). Os menores índices de diversidade $(\mathrm{H})$ e de equitabilidade $(\mathrm{E})$ para as espécies capturadas foram obtidos para a área de soja-milho em sistema de plantio convencional adjacente a povoamento de Pinus. Por outro lado, na área de fragmento florestal, as espécies de carabídeos e estafilinídeos capturadas apresentaram os maiores índices de diversidade e de equitabilidade. Esses resultados indicam que a comunidade desses besouros predadores, presentenaárea comculturacujosolo não foi movimentado para cultivo, apresentou-se melhor estruturada, istoé, com maior homogeneidade na abundância relativa dos indivíduos das espécies quando comparada à comunidade de carabídeos e estafilinídeos observada na área de plantio convencional, cujo solo recebeu procedimentos de aração e de gradeação antes da semeadura. As inferências do presente trabalho encontram sustentação em alguns autores (Stinner; House, 1990; CAIXeta et al., 2005), os quais relataram que sistemas de plantio direto favorecem a ocorrência de carabídeos, estafilinídeos e mesmo outros artrópodes por proporcionarem ambiente favorável ao desenvolvimento e reprodução desses organismos.

Tabela 1 - Número total de adultos de Carabidae e Staphylinidae capturados em povoamento dePinus eárea de cultivo anual em sistema de plantio convencional, índices de diversidade (H), equitabilidade (E), dominância (D), abundância (A), freqüência $(F)$ e constância $(C)$.

\begin{tabular}{|c|c|c|c|c|c|}
\hline Família / espécie & № de indivíduos & $\mathrm{D}$ & A & $\mathrm{F}$ & $\mathrm{C}$ \\
\hline \multicolumn{6}{|l|}{ Carabidae } \\
\hline Abaris basistriatus Chaudoir & 124 & sd & sa & sf & $\mathrm{w}$ \\
\hline Selenophorus alternans Dejean & 100 & $\mathrm{sd}$ & sa & sf & $\mathrm{w}$ \\
\hline Scarites sp. & 56 & $\mathrm{~d}$ & $\mathrm{ma}$ & $\mathrm{mf}$ & $\mathrm{w}$ \\
\hline Odontochila nodicornis (Dejean) & 34 & $\mathrm{~d}$ & $\mathrm{ma}$ & $\mathrm{mf}$ & $\mathrm{w}$ \\
\hline Megacephala sp. & 17 & $\mathrm{~d}$ & $\mathrm{ma}$ & $\mathrm{mf}$ & $\mathrm{y}$ \\
\hline Selenophorus seriatoporus Putz. & 15 & $\mathrm{~d}$ & $\mathrm{a}$ & $\mathrm{mf}$ & $\mathrm{y}$ \\
\hline Calosoma granulatum Perty & 12 & $\mathrm{~d}$ & c & $\mathrm{f}$ & $\mathrm{y}$ \\
\hline Galerita brasiliensis Dejean & 6 & nd & c & $\mathrm{f}$ & $\mathrm{y}$ \\
\hline Odontochila cupricollis Kollar & 4 & nd & c & $\mathrm{f}$ & $\mathrm{y}$ \\
\hline Loxandrus aff. subvittatus Straneo & 3 & nd & c & $\mathrm{f}$ & $\mathrm{z}$ \\
\hline Megacephala brasiliensis Kirby & 3 & nd & c & $\mathrm{f}$ & $\mathrm{z}$ \\
\hline Brachynus sp. & 1 & nd & $\mathrm{d}$ & $\mathrm{pf}$ & $\mathrm{z}$ \\
\hline Callida scutellaris Chaudour & 1 & nd & $\mathrm{d}$ & $\mathrm{pf}$ & $\mathrm{z}$ \\
\hline Eucheila sp. & 1 & nd & $\mathrm{d}$ & $\mathrm{pf}$ & $\mathrm{z}$ \\
\hline Lebia sp. & 1 & nd & $\mathrm{d}$ & pf & $\mathrm{z}$ \\
\hline Pseudabarys sp. & 1 & nd & $\mathrm{d}$ & pf & $\mathrm{z}$ \\
\hline Selenophorus sp. & 1 & nd & $\mathrm{d}$ & $\mathrm{pf}$ & $\mathrm{z}$ \\
\hline Sphalera plaumanni Liebre & 1 & nd & $\mathrm{d}$ & pf & $\mathrm{z}$ \\
\hline Outras espécies & 16 & - & - & - & - \\
\hline
\end{tabular}

Continua... 
Tabela 1 - Continuação

\begin{tabular}{|c|c|c|c|c|c|}
\hline Família / espécie & № de indivíduos & $\mathrm{D}$ & A & $\mathrm{F}$ & $\mathrm{C}$ \\
\hline \multicolumn{6}{|l|}{ Staphylinidae } \\
\hline Eulissus sp. & 2 & nd & $\mathrm{d}$ & $\mathrm{pf}$ & $\mathrm{z}$ \\
\hline Smilax pilosa (Fabricius) & 1 & nd & $\mathrm{d}$ & pf & $\mathrm{z}$ \\
\hline Xenopygus sp. & 1 & nd & $\mathrm{d}$ & pf & $\mathrm{z}$ \\
\hline Outras espécies & 4 & - & - & - & - \\
\hline Total de indivíduos & 405 & - & - & - & - \\
\hline Total de espécies & 29 & - & - & - & - \\
\hline $\mathrm{H}$ & 1,937 & - & - & - & - \\
\hline $\mathrm{E}$ & 0,636 & - & - & - & - \\
\hline
\end{tabular}

$\mathrm{sd}=$ super dominante, $\mathrm{d}=$ dominante, $\mathrm{nd}=$ não dominante, $\mathrm{sa}=$ super abundante, $\mathrm{ma}=$ muito abundante, $\mathrm{a}=$ abundante, $c=$ comum, $\mathrm{d}=$ dispersa, $\mathrm{sf}=$ super freqüente, $\mathrm{mf}=$ muito freqüente, $\mathrm{f}=$ freqüente, $\mathrm{pf}=$ pouco freqüente, $\mathrm{w}=$ constante, $\mathrm{y}=$ acessória, $\mathrm{z}=$ acidental

Tabela 2 - Número total de adultos de Carabidae e Staphylinidae capturados em fragmento florestal e área de cultivo anual em sistema de plantio direto, índices de diversidade $(H)$, equitabilidade (E), dominância (D), abundância (A), freqüência $(\mathrm{F})$ e constância $(\mathrm{C})$.

\begin{tabular}{|c|c|c|c|c|c|}
\hline Família / espécie & № de indivíduos & $\mathrm{D}$ & $\mathrm{A}$ & $\mathrm{F}$ & C \\
\hline \multicolumn{6}{|l|}{ Carabidae } \\
\hline Abaris basistriatus Chaudoir & 65 & $\mathrm{~d}$ & Ma & $\mathrm{mf}$ & $\mathrm{w}$ \\
\hline Odontochila nodicornis (Dejean) & 57 & $\mathrm{~d}$ & $\mathrm{Ma}$ & $\mathrm{mf}$ & w \\
\hline Scarites sp. & 52 & $\mathrm{~d}$ & $\mathrm{Ma}$ & $\mathrm{mf}$ & w \\
\hline Megacephala sp. & 33 & $\mathrm{~d}$ & $\mathrm{Ma}$ & $\mathrm{mf}$ & w \\
\hline Selenophorus seriatoporus Putz. & 30 & $\mathrm{~d}$ & Ma & $\mathrm{mf}$ & $\mathrm{w}$ \\
\hline Megacephala brasiliensis Kirby & 27 & $\mathrm{~d}$ & $\mathrm{Ma}$ & $\mathrm{mf}$ & $\mathrm{y}$ \\
\hline Selenophorus alternans Dejean & 10 & nd & $\mathrm{C}$ & $\mathrm{f}$ & $\mathrm{y}$ \\
\hline Calosoma granulatum Perty & 6 & nd & $\mathrm{D}$ & $\mathrm{pf}$ & $\mathrm{y}$ \\
\hline Odontochila cupricollis Kollar & 4 & nd & $\mathrm{D}$ & $\mathrm{pf}$ & $\mathrm{z}$ \\
\hline Pseudabarys sp. & 3 & nd & $\mathrm{R}$ & pf & $\mathrm{z}$ \\
\hline Sphalera plaumanni Liebre & 3 & nd & $\mathrm{R}$ & $\mathrm{pf}$ & $\mathrm{z}$ \\
\hline Selenophorus discopunctatus Dejean & 2 & nd & $\mathrm{R}$ & $\mathrm{pf}$ & $\mathrm{z}$ \\
\hline Selenophorus sp. & 2 & nd & $\mathrm{R}$ & $\mathrm{pf}$ & $\mathrm{z}$ \\
\hline Stratiotes sp. & 2 & nd & $\mathrm{R}$ & $\mathrm{pf}$ & $\mathrm{z}$ \\
\hline Arthrostictus speciosus (Dejean) & 1 & nd & $\mathrm{R}$ & $\mathrm{pf}$ & $\mathrm{z}$ \\
\hline Galerita brasiliensis Dejean & 1 & nd & $\mathrm{R}$ & $\mathrm{pf}$ & $\mathrm{z}$ \\
\hline Helluomorpha sp. & 1 & nd & $\mathrm{R}$ & $\mathrm{pf}$ & $\mathrm{z}$ \\
\hline Notobia amethystinus Dejean & 1 & nd & $\mathrm{C}$ & $\mathrm{f}$ & $\mathrm{z}$ \\
\hline Outras espécies & 7 & - & - & - & - \\
\hline Staphylinidae & & & & & \\
\hline Eulissus chalybaeus Mannerheim & 7 & nd & c & $\mathrm{f}$ & Y \\
\hline Glenus chrysis Gravenhorst & 7 & nd & $\mathrm{d}$ & $\mathrm{f}$ & Y \\
\hline Smilax pilosa (Fabricius) & 4 & nd & $\mathrm{d}$ & $\mathrm{pf}$ & Z \\
\hline Morfoespécie 1 & 1 & - & - & - & - \\
\hline Total de indivíduos & 326 & - & - & - & - \\
\hline Total de espécies & 27 & - & - & - & - \\
\hline $\mathrm{H}$ & 2,314 & - & - & - & - \\
\hline $\mathrm{E}$ & 0,760 & - & - & - & - \\
\hline
\end{tabular}

$\mathrm{d}=$ dominante, $\mathrm{nd}=$ não dominante, $\mathrm{ma}=$ muito abundante, $\mathrm{a}=$ abundante, $\mathrm{c}=$ comum, $\mathrm{d}=$ dispersa, $\mathrm{r}=\mathrm{rara}, \mathrm{mf}=$ muito freqüente, $\mathrm{f}=$ freqüente, $\mathrm{pf}=$ pouco freqüente, $\mathrm{w}=$ constante, $\mathrm{y}=$ acessória, $\mathrm{z}=$ acidental 
Tabela 3 - Modelos ajustados pelo método stepwise entre fatores meteorológicos e espécies dominantes de Carabidae capturadas em povoamento de Pinus e área de cultivo anual em sistema de plantio convencional.

\begin{tabular}{|c|c|c|c|c|c|c|}
\hline Espécie & Variável & $\begin{array}{c}\text { Estimativa dos } \\
\text { coeficientes }\end{array}$ & $\mathrm{R}^{2}$ parcial & $\mathrm{R}^{2}$ modelo & F Modelo & $\mathrm{P}$ \\
\hline \multirow{2}{*}{ Abaris basistriatus Chaudoir } & Intercepto & 0,885 & & 0,15 & 3,87 & \multirow{3}{*}{0,061} \\
\hline & Prec. pluvial & 0,038 & 0,15 & & & \\
\hline Selenophorus alternans Dejean & \multicolumn{5}{|c|}{ Nenhuma variável foi incluída no modelo } & \\
\hline & Intercepto & $-11,281$ & & 0,23 & 6,47 & \multirow{3}{*}{0,018} \\
\hline scarites sp. & Temp. mínima & 0,791 & 0,23 & & & \\
\hline Odontochila nodicornis (Deiean) & Intercepto & $-1,080$ & & 0,37 & 13,13 & \\
\hline & Prec. pluvial & 0,022 & 0,37 & & & 0,001 \\
\hline
\end{tabular}

$\mathrm{P}<0,05=$ significativo a $5 \%$ de probabilidade

$\mathrm{P}<0,01=$ significativo a $1 \%$ de probabilidade

$\mathrm{P}>0,05=$ não significativo a $5 \%$ de probabilidade

Tabela 4 - Modelos ajustados pelo método stepwise entre fatores meteorológicos e espécies dominantes de Carabidae capturadas em fragmento florestal e área de cultivo anual em sistema de plantio direto.

\begin{tabular}{|c|c|c|c|c|c|c|}
\hline Espécie & Variável & $\begin{array}{l}\text { Estimativa dos } \\
\text { coeficientes }\end{array}$ & $\mathrm{R}^{2}$ parcial & $\mathrm{R}^{2}$ modelo & F modelo & $\mathrm{P}$ \\
\hline \multirow{3}{*}{ Abaris basistriatus Chaudoir } & Intercepto & $-4,316$ & & 0,15 & 3,98 & \multirow{3}{*}{0,058} \\
\hline & Temp. mínima & 0,348 & 0,15 & & & \\
\hline & Intercepto & 1,237 & & 0,64 & 39,37 & \\
\hline \multirow[t]{2}{*}{ Odontochila nodicornis (Dejean) } & Prec. pluvial & 0,034 & 0,64 & & & \multirow[t]{2}{*}{0,0001} \\
\hline & Intercepto & $-2,404$ & & 0,42 & 16,16 & \\
\hline \multirow{2}{*}{ Megacephala sp. } & Prec. pluvial & 0,033 & 0,42 & & & \multirow[t]{2}{*}{0,001} \\
\hline & Intercepto & $-0,605$ & & 0,31 & 9,88 & \\
\hline Scarites sp. & Prec. Pluvial & 0,024 & 0,31 & \multirow[b]{2}{*}{0,14} & \multirow[b]{2}{*}{3,44} & 0,005 \\
\hline Selenophorus seriatoporus Putz. & $\begin{array}{l}\text { Intercepto } \\
\text { Temp. mínima }\end{array}$ & $\begin{array}{r}-3,117 \\
0,254\end{array}$ & 0,14 & & & 0,077 \\
\hline
\end{tabular}

$\mathrm{P}<0,05=$ significativo a $5 \%$ de probabilidade

$\mathrm{P}<0,01=$ significativo a $1 \%$ de probabilidade

$\mathrm{P}>0,05=$ não significativo a $5 \%$ de probabilidade

Entre as espécies amostradas nas duas áreas experimentais, seis especies foram dominantes (Tabelas 1 e 2). Abaris basistriatus Chaudoir, Odontochila nodicornis (Dejean) eScarites sp. foram dominantes nas duas áreas estudadas. Já Selenophorus alternans Dejean predominou apenas na área sob sistema de plantio convencional, enquanto Megacephala sp.e Selenophorusseriatoporus Putz. em sistema de plantio direto.Ellsbury etal.(1998) relataram que a determinação de espécies dominantes de insetos predadores tem sido considerada fundamental para o manejo de agentes de controle biológico natural, devido ao fato dessas espécies apresentarem potencial para utilização em programas de controle biológico de pragas. Portanto, as espécies dominantes observadas no presente estudo devem atuar como agentes de controle natural de insetos fitófagos, com potencial para serem aproveitadas em programas de controle biológico por conservação. 

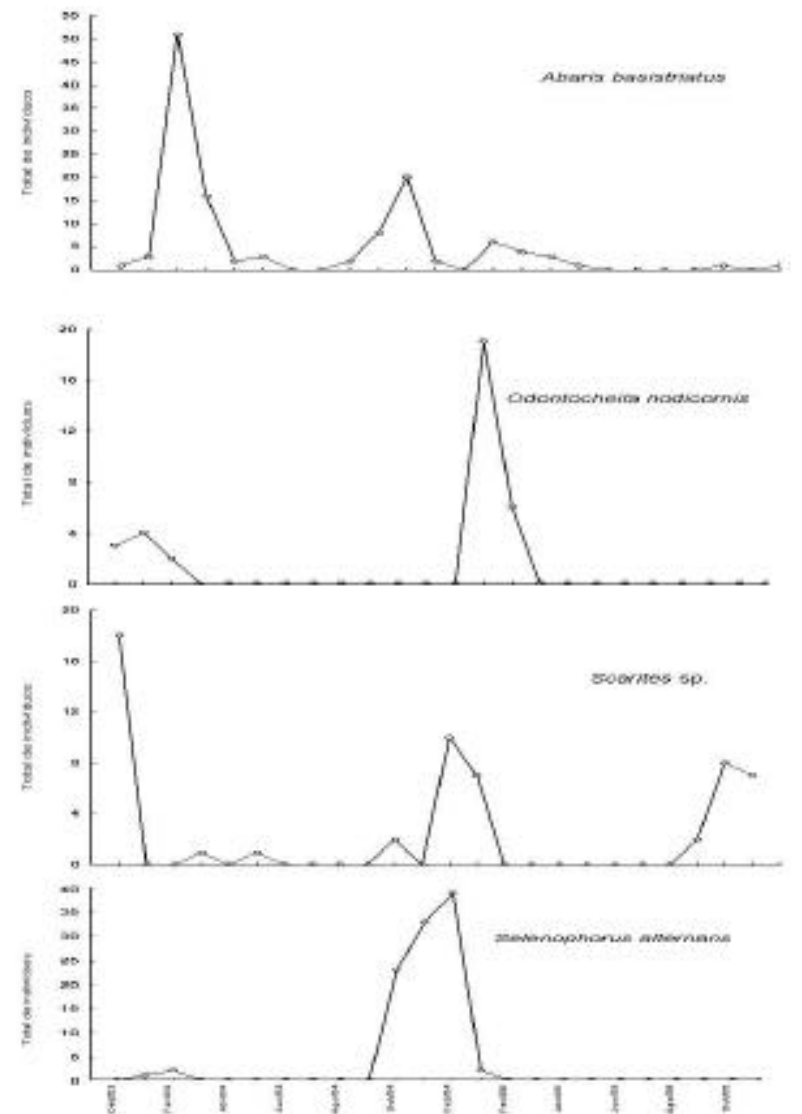

Fig. 1 - Flutuação populacional de carabídeos capturados em povoamento de Pinus e área de cultivo anual em sistema de plantio convencional.

Destaca-se que o carabídeo Calosoma granulatum Perty tem sido reconhecido como importante predador encontrado na cultura da soja (PASINI; Forester, 1994), mas no presente estudo não se classificou como espécie dominante. Ocultivo de milho na safra 2003/ 2004 e soja na safra 2004/2005 pode ser uma das razões para a constatação da baixa densidade da espécie, pois de acordo comPegoraro;FOERSTER(1988) a cultura do milho não constitui hábitat propício para C. granulatum.

Várias espécies de carabídeos e estafilinídeos foram capturadas em apenas uma das áreas. Apesar dessas espécies não terem sido abundantes, destacam-se os carabídeos Selenophorus discopunctatus Dejean e Stratiotes sp. e os estafilinídeos Eulissus chalybaeus Mannerheim e Glenuschrysis Gravenhorst ocorrendo apenas na área de fragmento florestal-cultura soja/ milho sob plantio direto, enquanto Loxandrus aff. subvittatus Straneo (Carabidae) e Eulissus sp. (Staphylinidae) foram observados no povoamento de Pinus-cultura soja/milho sob plantio convencional. Como citado anteriormente, a ocorrência de espécies decarabídeos nos hábitats mostra-se bastanteinfluenciada pelas condições de temperatura, luz, umidade
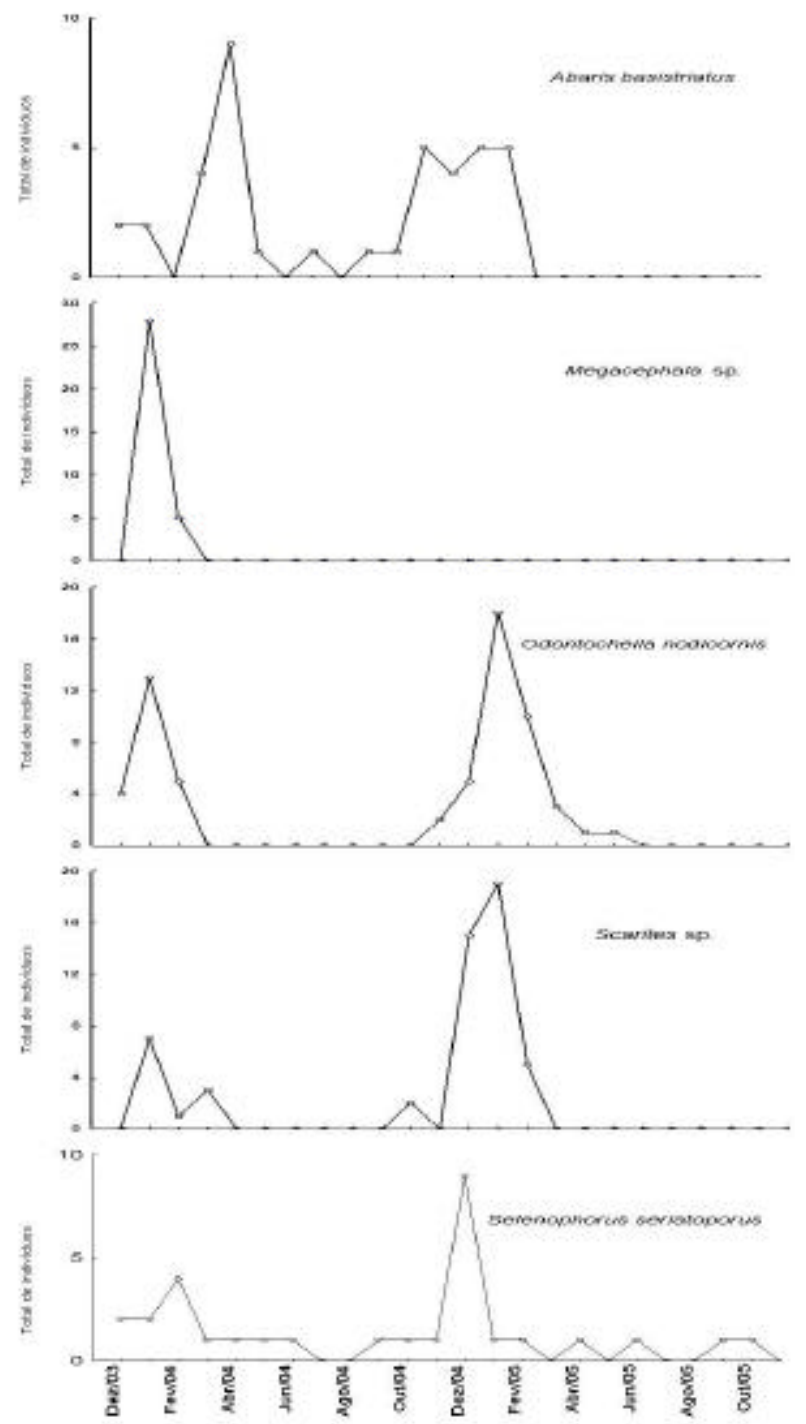

Fig. 2 - Flutuação populacional de carabídeos capturados em fragmento florestal eárea de cultivo anual em sistema de plantio direto.

do solo e cobertura vegetal (THIELE, 1977; LöveI; SunderLAND, 1996; KROMP, 1999). Portanto, a diferença observada na ocorrência de espécies se deve, provavelmente, às características de microclima, cobertura vegetal e cultivo do solo existentes em cada área, devendo evidenciar maior preferência das referidas espécies pelas condições abióticas e bióticas existentes nessas áreas.

De modo geral, as espécies dominantes apresentaram aumento de densidade populacional no período compreendido entre dezembro a janeiro (Figs. 1 e 2). Observação semelhante foi relatada por HoLtz et al. (2001) e FreITAs et al. (2002), ao registrarem maior pico populacional de carabideos nos meses de dezembro e janeiro em eucaliptais em Minas Gerais.

Dentre as variáveis meteorológicas analisadas, a precipitação pluvial foi a que mais influenciou na 
ocorrência de carabídeos (Tabelas 3 e 4). Esse fator explicou até $64 \%$ da variação observada na densidade populacional desses besouros. Pelos modelos obtidos, determinou-se que para valores da precipitação pluvial abaixo de 72,8 mm não foram capturados indivíduos de Megacephalasp., o mesmo ocorrendo comO. nodicornise Scaritessp. para quantidades de precipitação de 36,4 a $49,1 \mathrm{~mm}$ e $25,2 \mathrm{~mm}$, respectivamente. Esses resultados sugerem que $O$. nodicornis e, principalmente, Megacephalasp. foram as espécies mais exigentes quanto à umidade do solo para se deslocarem. Deve ser ressaltado que a umidade do solo representa um dos mais importantes fatores influenciando o deslocamento de carabídeos sobre ele. A precipitação pluvial pode proporcionar níveis adequados de umidade no solo permitindo aos adultos se movimentarem sobre ele (Thomaset al., 2002). Em plantios de eucalipto na Amazônia, PinTo et al. (2004) também verificaram que o maior número de indivíduos e de espécies de Carabidae foram coletados nos meses em que se registraram os maiores índices pluviais, principalmente no mês de janeiro, indicando relação direta entre ocorrência das espécies desses besouros com períodos de chuva.

A temperatura mínima atuou positiva e significativamente sobre a densidade populacional do carabídeo Scarites sp. em área sob sistema de plantio convencional, explicando $23 \%$ da ocorrência da espécie nos hábitats (Tabela 3 ). O modelo obtido permite determinar que adultos da espécie fossem capturados nas armadilhas apenas quando a temperatura mínima esteve acima de $14,3^{\circ} \mathrm{C}$.

Os resultados do presente estudo indicaram que os carabídeos foram mais abundantes e apresentaram maior riqueza de espécies que os estafilinídeos estando a comunidade desses besouros melhor estruturada em área sob sistema de plantio direto. As populações dos carabídeos foram mais numerosas entre dezembro a janeiro e mostraram-se influenciadas principalmente pela precipitação pluvial, concordando com THOMAset al. (2002), segundo os quais a umidade do solo está diretamente relacionada com a precipitação pluvial representando um dos mais importantes fatores que atuam sobre a atividade de carabídeos no solo.

\section{AGRADECIMENTOS}

Ao Prof. Dr. José Carlos Barbosa, Depto. de Ciências Exatas da FCAV/Unesp, pelo auxílio nas análises estatísticas e ao Dr. Sérgio Ide, Instituto Biológico/Apta, pela identificação de Carabidae e Staphylinidae.

\section{REFERÊNCIAS}

ASTERAKI, E.J.; HANKS, C.B.; CLEMENTS, R.O. The influence of different types of grassland field margin on carabid beetle (Coleoptera, Carabidae) communities. Agriculture, Ecosystem E Environment, v.54, n.3, p.195-202, 1995.

BARBOSA, M.G.V. da; FONSECA, C.R.V. da. Coleopterofauna visitante de Theobroma grandiflorum Schum. (Sterculiaceae) de uma plantação nos arredores de Manaus, Amazonas, Brasil. Acta Amazonica, v.32, n.1, p.83-100, 2002.

BARROS, R.; DEGRANDE, P.E.; RIBEIRO, J.F.; RODRIGUES, A.L.L.; NOGUEIRA, R.F.; FERNANDES, M.G. Flutuação populacional de insetos predadores associados a pragas do algodoeiro. Arquivos do Instituto Biológico, São Paulo, v.73, n.1, p.57-64, 2006.

BEDFORD, S.E.; UsHER, M. B. Distribution of arthropod species across the margins of farm woodlands.

Agriculture, Ecosystem \& Environment, Amsterdam, v.48, p.295-305, 1994.

CAIXETA, D.F.; NOLASCO, L.A.; BARRIGOSSI, J.A.F.; QUINTELA, E.D.; MOREIRA, J.A.A.; DIDONET, A. Diversidade de artrópodes de solo cultivado com feijoeiro em sistemas de manejo de dolos e coberturas de inverno. 2005. Disponível em: <http:/ / www.cnpaf. embrapa.br/conafe2005-0331.pdf>. Acesso em: 31 ago. 2007.

CIVIDANES, F.J.; ATHAYDE, M.L.F.; SABUGOSA, E.T. Levantamento populacional de artrópodes associados a cultivares de soja semeadas em diferentes densidades. Revista de Agricultura, v.71, n.2, p.243-250, 1996.

CIVIDANES, F.J.; SOUZA,V. de P.; SAKEMI, L.K. Composição faunística de insetos predadores em fragmento florestal e em área de hortaliçcas na região de Jaboticabal, Estado de São Paulo. Acta-Scientiarum, v. 25, n.2, p.315-321, 2003.

CLARK, M.S.; LUNA, J.M.; STONE, N.D.; YOUNGMAN, R.R. Generalist predator consumption of armyworm (Lepidoptera: Noctuidae) and effect of predator removal on damage in no-till corn. Environmental Entomology, v.23, n.3, p.617-622, 1994.

DENT, D. (Ed.). Insect pest management. Wallingford: CAB International, 1991. 604p.

DIDONET, J.; FRAGOSO, D. de B.; PELUZIO, J.M.; SANTOS, G.R. dos Flutuação populacional de pragas e seus inimigos naturais em soja no Projeto Rio Formoso, Formoso do Araguaia, TO, Brasil. Acta Amazônica, v.28, n.1, p.67-74, 1998.

DRAPER, N.R.; SMITH, H. (Ed.). Applied regression analysis. 2.ed. New York: John Wiley, 1981. 709p. 
ELLSBURY, M.M.; PIKUL JUNIOR J.L.; WOODSON, W.D. A review of insect survival in frozen soils with particular reference to soil-dwelling stages of corn rootworms. Cold Region Science and Technology, v.27, p.49-56, 1998.

FREITAS, F.A. de; ZANUNCIO, T.V.; LACERDA, M.C.; ZANUNCIO, J.C. Fauna de Coleoptera coletada com armadilhas luminosas em plantios de Eucaliptus grandis em Santa Bárbara, Minas Gerais. Revista Árvore, v.26, n.4, p.505-511, 2002.

GALLI, J. C.; RAMPAZZO, E. F. Enemigos naturales predadores de Anastrepha (Diptera, Tephritidae) capturados con trampas de suelo en huertos de Pisidium guajava L. Boletin de Sanidad Vegetal Plagas, v.22, n.2, p.297-300, 1996.

HOLLAND, J. M.; LUFF, M. L. The effects of agricultural practices on Carabidae in temperate agroecosystems. Integrated Pest Management Reviews, v.5, n.2, p.109-129, 2000.

HOLLAND, J.M. Carabid beetles: their ecology, survival and use in agroecosystems. In: HOLLAND, J.M. (Ed.). The agroecology of carabid beetles. Andover: Intercept, 2002. 356p.

HOLTZ, A.M.; ZANUNCIO, T.V.; ZANUNCIO, J.C.; PRATISSOLI, D. Coleópteros coletados em plantio de Eucaliptus urophylla na região de Três Marias, Minas Gerais. Revista Floresta, v.31, n.1/2, p.32-41, 2001.

KROMP, B. Carabid beetles in sustainable agriculture: a review on pest control efficacy, cultivation impacts and enhancement. Agriculture, Ecosystem \& Environment, v.74, n.1/3, p.187-228, 1999.

LÖVEI, G.L.; SUNDERLAND, K.D. Ecology and behavior of ground beetles (Coleoptera: Carabidae). Annual Review of Entomology, v.41, p.231-256, 1996.

LUFF, M.L. Use of carabids as environmental indicators in grasslands and cereals. Annales Zoologici Fennici, v.33, p.185-195, 1996.

LYS, J.A.; NENTWIG, W. Surface activity of carabid beetles inhabiting cereal fields. Seasonal phenology and the influence of farming operations on five abundant species. Pedobiologia, v.35, p.129-138, 1991.

MENALLED, F.D.; LEE. J.C.; LANDIS, D.A.

Manipulating carabid beetle abundance alters prey removal rate in corn fields. Biocontrol, v.43, p.441-456, 1999.
PASINI, A.; FOERSTER, L.A. Efeito de inseticidas sobre Calosoma granulatum P. (Col.: Carabidae). Anais da Sociedade Brasileira de Entomologia, v.23, p.455-460, 1994.

PEGORARO, R.A.; FOERSTER, L.A. Abundância e distribuição de larvas e adultos de Calosoma granulatum Perty, 1830 (Coleoptera: Carabidae) dentre cultivares de soja em diferentes épocas de semeadura. Anais da Sociedade Brasileira de Entomologia, v.17, p.237-248, 1988.

PFIFFNER, L.; LUKA, H. Overwintering of arthropods in soils of arable fields and adjacent semi-natural habitats. Agriculture, Ecosystem \& Environment, v.78, n.3, p.215-222, 2000.

PINTO, R.; ZANUNCIO JUNIOR, J.S.; ZANUNCIO, T.V.; ZANUNCIO, J.C.; LACERDA, M.C. Coleópteros coletados com armadilhas luminosas em plantios de Eucaliptus urophylla na região Amazônica Brasileira. Ciência Florestal, v.14, n.1, p.111-119, 2004.

SILVEIRA NETO, S.; MONTEIRO, R.C.; ZUCCHI, R.A.; MORAES, R.C.B. de. Uso da análise faunística de insetos na avaliação do impacto ambiental. Scientia Agricola, v.52, n.9/15, p.9-15, 1995.

SOTHERTON, N.W. The distribution and abundance of predatory arthropods overwintering on farmland. Annals of Applied Biology, v.105, n.3, p.423-429, 1984.

STINNER, B.R.; HOUSE, G.J. Arthropods and other invertebrates in conservation-tillage agriculture. Annual Review of Entomology, v.35, p.299-318, 1990.

THIELE, H.U. Carabid beetles in their environments. Berlin: Springer, 1977. 369p.

THOMAS, C.F.G.; HOLLAND, J.M.; BROWN, N.J. The spatial distribution of carabid beetles in agricultural landscapes. In: Holland, J.M. (Ed.). The agroecology of carabid beetles. Andover: Intercept, 2002. 356p.

THOMAZINI, M.J.; THOMAZINI, A.P.B.W. Pragas e inimigos naturais associados à cultura da soja no Estado do Acre. Rio Branco: EMBRAPA-Acre, 2001. 22p. (Boletim de Pesquisa, n.32).

ZANUNCIO, J.C.; BRAGANÇA, M.A.L.; LARANJEIRO, A.J.; FAGUNDES, M. Coleópteros associados à eucaliptocultura nas regiões de São Mateus e Aracruz, Espírito Santo. Revista Ceres, v.40, p.584-590, 1993.

Recebido em 18/9/07

Aceito em 3/9/08 